\title{
Relationship between panic disorder and plasma neuropeptide-S level
}

\author{
Hayriye Baykan ${ }^{1}$, Özgür Baykan ${ }^{2}$, Emre C. Esen ${ }^{1}$, Hayrettin Kara³, Adnan A. HişmioĞullari ${ }^{3}$, \\ TUNAY KARLIDERE ${ }^{1}$
}

${ }^{1}$ Balikesir University Medical School Psychiatry Department, Balikesir, Turkey.

${ }^{2}$ Balikesir Atatürk Public Hospital Biochemistry Laboratory, Balikesir, Turkey.

${ }^{3}$ Balikesir University Medical School Biochemistry Department, Balikesir, Turkey.

Received: 12/13/17 - Accepted: 07/24/18

DOl: 10.1590/0101-60830000000163

\begin{abstract}
Background: Panic disorder has long been associated with the changes in various neurotransmitters, such as Neuropeptide-S (NPS). Objective: In this study we aimed to determine whether there is a relationship between blood NPS levels and panic disorder. Methods: Twenty nine patients with panic disorder and thirty two healthy control subjects who were age and gender matched were enrolled to the study. Blood samples were taken from participants and plasma NPS levels were quantified by using an ELISA kit. Results: In the study group, median NPS blood level was $16.7 \mathrm{pg} / \mathrm{mL}$ and in the control group it was $32.5 \mathrm{pg} / \mathrm{mL}$. There was a statistically significant difference $(\mathrm{p}=0.021)$. Using receiver operating characteristics (ROC) curve, sensitivity and specificity of NPS blood level, for diagnosing panic disorder was calculated, and it was found 79.3\% and 56.25\% respectively (AUC:0.672, 95\% CI: 0.540-0.787). Discussion: Malfunction at the NPS modulatory system in the cortical areas (which is causing excitations in brain areas, such as amygdala and hypothalamus) does not only increase anxiety symptoms and risk of panic disorder but also causes panic disorder patients to have lower plasma NPS levels than the control group. Therefore it can be argued that such malfunction can be treated with a systemic treatment.
\end{abstract}

Baykan H et al. / Arch Clin Psychiatry. 2018;45(4):79-81

Keywords: Panic disorder, neuropeptide-S, anxiety disorder.

\section{Introduction}

A panic attack is a sudden and unexpected rush of immense anxiety. It has a lifetime prevalence of $13.1 \%^{1}$. Not every patient who had a panic attack can be diagnosed with panic disorder. According to Diagnostic and Statistical Manual; a patient experiencing panic attacks can only be diagnosed as the panic disorder if that person is experiencing persistent anxiety about having more panic attacks and/or if these attacks are causing significant maladaptive changes in the behavior. Although two-thirds of the people who have panic attacks, experience them recurrently; only around one-sixth of them are eligible for panic disorder diagnosis ${ }^{1}$.

Panic disorder has long been associated with various neurotransmitter signaling changes. In brief, there is a reduction of inhibitory signaling in the central nervous system among panic disorder patients. This postulate is backed up by the studies showing an increased function in glutamatergic neurons and decreased function in GABAergic neurons. There are also other monoamines (serotonin and noradrenaline) and neuropeptides (cholecystokinin) which have been associated with panic disorder as well2.

Neuropeptide-S (NPS) was discovered around two decades ago. NPS has been shown to have several physiological roles; such as sleep regulation, modulation of feeding behavior, activation of the hypothalamic-pituitary-adrenal axis and adrenocorticotropic hormone $(\mathrm{ACTH})$ release; extinguishing anxiety and conditioned fear responses ${ }^{3-5}$.

Animal studies have shown that intranasal6-8 and intracerebroventricular ${ }^{7-10}$ NPS injections were able to reduce symptoms associated with anxiety and panic disorder. Also, Neuropeptide-S Receptor (NPSR) polymorphism has been shown to increase the risk of panic disorder both in animal and human studies ${ }^{3-5,10-18}$. However, there has been no study that examines the relationship between blood NPS levels and panic disorder up till now.

In this study, we aimed to determine whether there is a relationship between blood NPS levels and panic disorder. To examine this hypothesis, we quantified the plasma NPS levels of panic disorder patients and healthy controls.

\section{Methods}

\section{Subjects}

We have designed our research as a case-control study. After getting an ethics committee approval we have prospectively enrolled participants to our study from January 2015 until December 2015. All the patients admitted to the Balikesir University Psychiatry outpatient service during 2015, who were diagnosed with panic disorder (either with or without agoraphobia) for the first time and who were not using any psychotropic or beta blocker drugs at the time of their admission, were asked to enroll to our study.

All the subjects, both the participants of study and control group, undergone structured interview of SCID (Structured Clinical Interview for DSM-IV). Medical history of all the participants were self declared. Their drug history were verified with the Republic of Turkey Social Security Institution's online pharmaceutical database.

Participants of the study group were excluded if they were given any other psychiatric diagnosis other than panic disorder. Additionally, participants of the control group were excluded from the study if any psychiatric diagnosis was given. Using any psychotropic or beta blocker drug was an exclusion criterion for all subjects.

Twenty-nine panic disorder patients who met criteria mentioned above agreed to participate in our study and gave a written informed consent. Thirty-two, age and gender matched healthy volunteers were enrolled to the study for the control group. This research was approved by the Balikesir University School of Medicine Clinical Research Ethics Committee on 10/01/2014 with the approval number 2014/03.

\section{Psychiatric scales}

All participants were asked to fill the Panic and Agoraphobia Scale (PAS). PAS is a self-rated Likert type scale in which total scores can vary between 0 and 52 . PAS measures panic attacks, 
agoraphobia, anticipatory anxiety, disability, and worries among panic attack patients, either with or without agoraphobia. Even though PAS is not intended as a diagnostic instrument, it is a convenient tool to separate panic disorder patients from healthy individuals. Validity and reliability of the Turkish version of PAS has been established ${ }^{19}$.

\section{Blood samples}

Blood samples were taken from participants to determine the blood level of NPS. Fasting venous blood samples were extracted and were collected in EDTA tubes (Becton, Dickinson, USA). Samples were centrifuged at $1300 \times \mathrm{g}$ for 10 minutes for plasma separation. Plasma samples and $1 \%$ concentrated protease inhibitor cocktail (Sigma Aldrich product number: P8340) were mixed in Eppendorf tubes. These mixtures were stored at $-20^{\circ} \mathrm{C}$ while waiting for the further processing. NPS was quantified by using a commercial Human NPS (Neuropeptide S) ELISA kit (Wuhan Fine Biological Technology Company Ltd., China).

\section{Statistics}

All statistic analyses were made on SPSS version 15.0 (SPSS, Chicago, IL, USA). To determine the sensitivity and specificity of the blood level of NPS for diagnosing panic disorder ROC curve was made an area under the curve was calculated.

\section{Results}

Mean age of the study and control groups were $38.6 \pm 9.0$ years and $39.3 \pm 9.3$ years respectively. There was no statistically significant difference between the two groups $(\mathrm{p}=0.768)$.

The panic disorder group consisted $71.9 \%$ of females whereas control group consisted $86.2 \%$ of females. There was no statistically significant difference regarding the gender between two groups $(\mathrm{p}=0,172)$.

In the panic disorder group, median NPS plasma level was 16.7 $\mathrm{pg} / \mathrm{mL}$, with a minimum of 3.7 and a maximum of 99.3 . In the control group, median NPS blood level was $32.5 \mathrm{pg} / \mathrm{mL}$, with a minimum of 3 and maximum of 156.1. A statistically significant difference was observed between the two groups $(\mathrm{p}=0.021)$ (Figure 1). There was not a statistically significant correlation between PAS scores and blood NPS levels ( $r s=-0.232, p=0.072)$. Optimal cut off score for NPS blood level was found 18.28 with the receiver operating characteristics (ROC) curve. Using that cut-off score; sensitivity and specificity of NPS blood level were calculated for diagnosing panic disorder. The results were $79.3 \%$ and $56.25 \%$ respectively (AUC:0.672, 95\% CI: 0.540-0.787) (Figure 2).

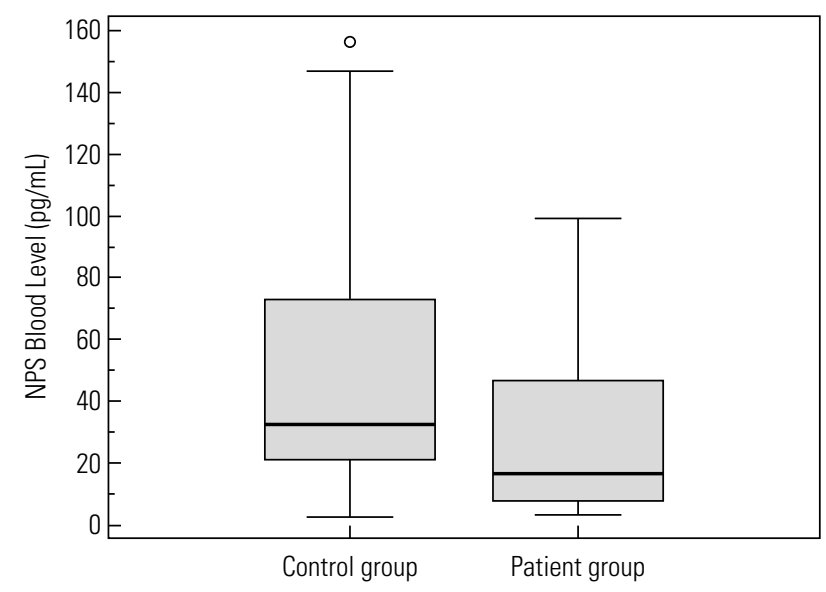

Figure 1. Medium NPS blood levels in patient and control groups.

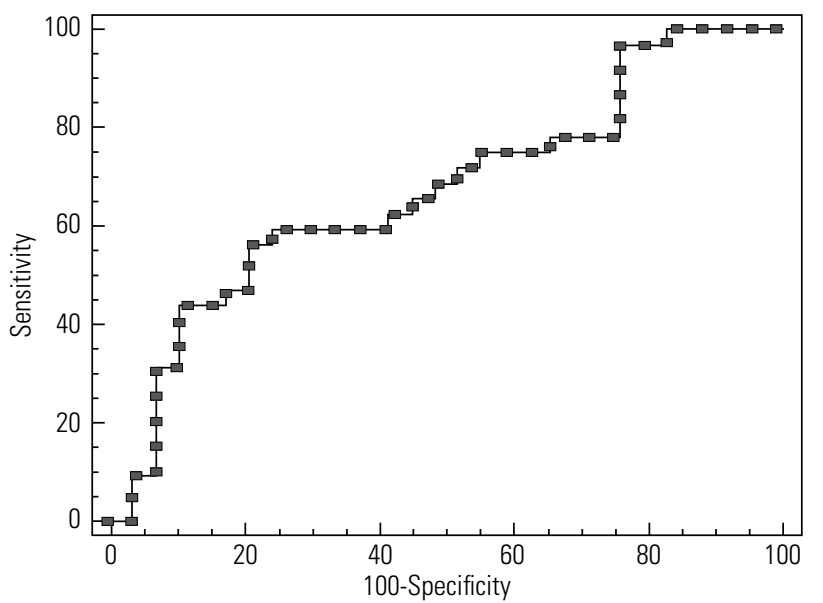

Figure 2. ROC curve of sensitivity and 100-specificity of NPS blood level for diagnosis of panic disorder.

\section{Discussion}

NPS is a, recently discovered, neurotransmitter which consists of 20 amino acids. Since NPS has been uncovered, it has been associated with different physiological systems and attributed to various functions such as sleep regulation, modulation of feeding behavior, activation of the hypothalamic-pituitary-adrenal axis and release of adrenocorticotropic hormone (ACTH); extinguishing anxiety and conditioned fear responses ${ }^{3-5}$. Even though expression of NPS is mostly limited to brainstem areas, mRNA of the NPS receptor (NPSR) has been demonstrated to be widely expressed in various brain regions, including olfactory regions and amygdala, hippocampal formation, and hypothalamus ${ }^{4}$.

At the first glance, the data on the relationship between NPS and panic disorder can give the impression that it is contradictory. On the one hand, NPS has crucial functions in extinguishing anxiety and conditioned fear responses ${ }^{3-5}$, and administration of NPS (both intranasally and intracerebroventricularly) has anxiolytic properties $^{6-10}$. On the other hand, NPS has been shown to activate hypothalamic-pituitary-adrenal axis and increases the release of $\mathrm{ACTH}^{3-5}$ and mutations that make NPSR more active, for both single nucleotide polymorphisms and mutations at the promoter sequence, causes subjects to be more susceptible to have panic disorder ${ }^{3-5,10-17}$.

The modulatory function of the NPS and NPSR explains the findings mentioned in the previous paragraph ${ }^{3-5}$. NPSR is mainly found on glutamatergic neurons, which are excitatory. However, these glutamatergic neurons mostly innervate GABAergic interneurons, especially in the amygdala. Overall NPSR activation causes inhibition in brain areas such as the amygdala. Therefore NPS has a modulatory role rather than a straightforward inhibitory or excitatory function ${ }^{3-5,10,11}$.

The neuroimaging studies support the discussions mentioned above. Human neuroimaging studies done by fMRI demonstrated that subjects who carried a mutated T allele for the rs324981 polymorphism in NPSR-1 gene showed increased activity in especially amygdala and prefrontal cortex. This increased activity is thought to cause a higher risk for anxiety and panic disorders ${ }^{13-18}$.

So far there has not been any study which investigated the relationship between plasma NPS levels and panic disorder. Most of the human studies on the subject looked into polymorphisms in the genes which are coding NPS and NPSR. Moreover, animal studies quantified NPS and NPSR mRNA in the brain tissue of mice and rats, on top of investigating similar polymorphisms mentioned earlier. However, our study brings forward a new approach to the subject.

Our hypothesis was, there would be a statistically significant difference in plasma NPS levels between panic disorder and control group. As hypothesized, possibly due to a malfunction at the NPS 
modulatory system in the cortical areas, panic disorder patients had lower plasma NPS levels than the control group. It can be argued that such malfunction can be treated with a systemic treatment. Hence intranasal administration of NPS was found to have anxiolytic properties, as it was mentioned previously.

Although there was a statistically significant difference between two groups in plasma NPS level, there was no relevant relationship between the PAS scores and blood NPS levels. These results can be caused by the size of our study. Moreover, PAS has five sub-scores but a total of 14 questions, which decreases the reliability of the test and caused the results reached.

Major limitation of our study was our small sample size which may have resulted in decreased statistical significance in our results. Moreover, small sample size limited our ability to obtain gender specific results. Although among female participants there was a statistically significant difference of plasma NPS levels between subject and control groups, there was no such difference among male participants. From the total of 61 participants, only 13 being men may have caused this result.

Furthermore, one blood sample was taken at the first admission to our outpatient service. Obtaining more samples from participants, including after sufficient treatment, can give us a better opinion on the trajectory of NPS throughout the progress and resolution of the disorder. Even though PAS is an established measurement of various sub-scales mentioned in the methods section; future researches can enhance study the design by including other panic or anxiety scales, such as Hamilton Anxiety Rating Scale and Panic Disorder Severity Scale.

In conclusion, NPS plasma levels of the panic disorder patients are found to be significantly lower than the control group. This result can be interpreted as a possible malfunction at the modulatory NPS system in panic disorder patients which causes NPS levels to be lower than the healthy population. Impaired NPS production and therefore function are producing excitations in brain areas, such as the amygdala, which results in anxiety symptoms and increased risk of panic disorder. Our findings are in line with the previous studies and take us a step closer to comprehending the roles of NPS in panic disorder and its possible use for the treatment of the disease.

\section{Acknowledgements}

This research has been supported by Balikesir University Scientific Research Projects Coordination Unit. Project Number: 71, Year: 2014.

Parts of this research has been presented at the 53. Turkish National Psychiatry Congress on 6th of October 2017.

\section{References}

1. de Jonge P, Roest AM, Lim CC, Florescu SE, Bromet EJ, Stein DJ. Cross-national epidemiology of panic disorder and panic attacks in the world mental health surveys. Depress Anxiety. 2016;33(12):1155-77.

2. Martin EI, Ressler KJ, Binder E, Nemeroff CB. The neurobiology of anxiety disorders: brain imaging, genetics, and psychoneuroendocrinology. Psychiatr Clin North Am. 2009;32(3):549-75.
3. Reinscheid RK. Neuropeptide S: anatomy, pharmacology, genetics and physiological functions. Results Probl Cell Differ. 2008;46:145-58.

4. Ghazal P. The Physio-Pharmacological Role of the NPS/NPSR System in Psychiatric Disorders: A Translational Overview. Curr Protein Pept Sci. 2016;17(4):380-97.

5. Pape HC, Jüngling K, Seidenbecher T, Lesting J, Reinscheid RK. Neuropeptide S: a transmitter system in the brain regulating fear and anxiety. Neuropharmacology. 2010;58(1):29-34.

6. Ionescu IA, Dine J, Yen YC, Buell DR, Herrmann L, Holsboer F, et al. Intranasally administered neuropeptide S (NPS) exerts anxiolytic effects following internalization into NPS receptor-expressing neurons. Neuropsychopharmacology. 2012;37(6):1323-37.

7. Lukas M, Neumann ID. Nasal application of neuropeptide $S$ reduces anxiety and prolongs memory in rats: social versus non-social effects. Neuropharmacology. 2012;62(1):398-405.

8. Dine J, Ionescu IA, Stepan J, Yen YC, Holsboer F, Landgraf R. Identification of a role for the ventral hippocampus in neuropeptide S-elicited anxiolysis. PLoS One. 2013;8(3):e60219.

9. Pulga A, Ruzza C, Rizzi A, Guerrini R, Calo G. Anxiolytic- and panicolytic-like effects of Neuropeptide $S$ in the mouse elevated T-maze. Eur J Neurosci. 2012;36(11):3531-7.

10. Slattery DA, Naik RR, Grund T, Yen YC, Sartori SB, Füchsl A, et al. Selective breeding for high anxiety introduces a synonymous SNP that increases neuropeptide S receptor activity. J Neurosci. 2015;35(11):4599-613.

11. Donner J, Haapakoski R, Ezer S, Melén E, Pirkola S, Gratacòs M, et al. Assessment of the neuropeptide $S$ system in anxiety disorders. Biol Psychiatry. 2010;68(5):474-83.

12. Okamura N, Hashimoto K, Iyo M, Shimizu E, Dempfle A, Friedel S, et al. Gender-specific association of a functional coding polymorphism in the Neuropeptide $S$ receptor gene with panic disorder but not with schizophrenia or attention-deficit/hyperactivity disorder. Prog Neuropsychopharmacol Biol Psychiatry. 2007;31(7):1444-8.

13. Raczka KA, Gartmann N, Mechias ML, Reif A, Büchel C, Deckert J, et al. A neuropeptide $S$ receptor variant associated with overinterpretation of fear reactions: a potential neurogenetic basis for catastrophizing. Mol Psychiatry. 2010;15(11):1045, 1067-74.

14. Domschke K, Reif A, Weber H, Richter J, Hohoff C, Ohrmann P, et al. Neuropeptide $S$ receptor gene -- converging evidence for a role in panic disorder. Mol Psychiatry. 2011;16(9):938-48.

15. Dannlowski U, Kugel H, Franke F, Stuhrmann A, Hohoff C, Zwanzger P, et al. (Neuropeptide-S (NPS) receptor genotype modulates basolateral amygdala responsiveness to aversive stimuli. Neuropsychopharmacology. 2011;36(9):1879-85.

16. Guhn A, Domschke K, Müller LD, Dresler T, Eff F, Kopf J, et al. Neuropeptide $S$ receptor gene variation and neural correlates of cognitive emotion regulation. Soc Cogn Affect Neurosci. 2015 Dec;10(12):1730-7.

17. Ruland T, Domschke K, Schütte V, Zavorotnyy M, Kugel H, Notzon S. Neuropeptide $S$ receptor gene variation modulates anterior cingulate cortex Glx levels during CCK-4 induced panic. Eur Neuropsychopharmacol. 2015;25(10):1677-82.

18. Domschke K, Akhrif A, Romanos M, Bajer C, Mainusch M, Winkelmann J, et al. Neuropeptide S Receptor Gene Variation Differentially Modulates Fronto-Limbic Effective Connectivity in Childhood and Adolescence. Cereb Cortex. 2017;27(1):554-66.

19. Tural U, Fidaner H, Alkin T, Bandelow B. Validity and reliability of the Turkish version of the Panic and Agoraphobia Scale. Turk Psikiyatri Derg. 2000;11:29-39. 